\title{
Artesunate overcomes drug resistance in multiple myeloma by inducing mitochondrial stress and non-caspase apoptosis
}

\author{
Xenofon Papanikolaou ${ }^{1}$, Sarah Johnson ${ }^{1}$, Tarun Garg ${ }^{1}$, Erming Tian', Ruslana \\ Tytarenko$^{1}$, Qing Zhang ${ }^{1}$, Caleb Stein ${ }^{1}$, Bart Barlogie ${ }^{1}$, Joshua Epstein ${ }^{1}$ and \\ Christoph Heuck ${ }^{1}$ \\ ${ }^{1}$ Myeloma Institute for Research and Therapy, University of Arkansas for Medical Sciences, Little Rock, AR
}

Correspondence to: Christoph Heuck, email: CJHeuck@uams.edu

Keywords: artesunate, apoptosis, caspases, myeloma, ROS

Received: February 18, $2014 \quad$ Accepted: March 22, $2014 \quad$ Published: March 24, 2014

This is an open-access article distributed under the terms of the Creative Commons Attribution License, which permits unrestricted use, distribution, and reproduction in any medium, provided the original author and source are credited.

\section{ABSTRACT:}

Although novel drugs have contributed immensely to improving outcomes of patients with multiple myeloma (MM), many patients develop drug resistance and ultimately succumb to MM. Here, we show that artesunate, an anti-malarial drug, reliably induces cell death in vitro in naïve as well as drug-resistant MM cells at concentrations shown to be safe in humans. Artesunate induced apoptosis predominantly through the non-caspase mediated pathway by primarily targeting mitochondria and causing outer mitochondrial membrane permeabilization that led to cytosolic and subsequent nuclear translocation of mitochondrial proteins apoptosis inducing factor (AIF) and endonuclease G (EndoG). Nuclear translocation of AIF and EndoG was accompanied by low levels of reactive oxygen species (ROS) and increased mitochondrial production of superoxide. These effects were present before apoptosis was evident and were related to intracellular levels of bivalent iron $\left(\mathrm{Fe}^{+2}\right)$. Artesunate's unique mechanism probably was at least partially responsible for, its ability to act synergistically with multiple anti-myeloma agents. Our findings suggest that artesunate acts through iron to affect the mitochondria and induce low ROS and non-caspase-mediated apoptosis. Its potency, toxicity profile, and synergism with other drugs make it an intriguing new candidate for MM treatment.

\section{INTRODUCTION}

With therapeutic advances over the last two decades, 5-year life expectancy for patients diagnosed with multiple myeloma (MM) has increased from $15 \%$ [1] to $70 \%$ [2]. Nevertheless, treatment of patients diagnosed with high-risk MM [3] or those who relapse after exposure to a multitude of anti-MM agents remains a significant challenge. For instance, Kumar et al. recently showed that MM patients who developed resistance to bortezomib (BZ) and IMiDs have an ominous median survival prognosis of only 9 months [4]. This patient population is in clear need of new drugs without cross-resistance to existing agents and, ideally, that function through a mechanism that has not yet been exploited for therapy.

Recent research has demonstrated that artemisinbased endoperoxide drugs can function as experimental cancer chemotherapeutics with significant activity in cell- based and animal studies [5-8]. These drugs constitute an important class of FDA-approved antimalarial agents, of which artesunate (ART) is the most potent available today [9]. ART's clinically proven favorable toxicity profile for malaria treatment [9], combined with its potential as a cancer chemotherapeutic, prompted us to examine its in vitro anti-MM activity.

In this study we establish the in-vitro effectiveness of ART against MM and in MM models of BZ resistance. Furthermore, we show that ART induces apoptosis mainly through the non-caspase mediated pathway, primarily targeting mitochondria, and that the effect is related to intracellular levels of bivalent iron. MM cells' "commitment" to apoptosis after treatment with ART is characterized by low cellular levels of reactive oxygen species (ROS). These unconventional mechanisms of action could help explain the absence of crossresistance to ART in MM models of in-vitro established 
chemoresistance and the synergism between ART and different frontline anti-myeloma agents.

\section{RESULTS}

\section{ART inhibits viability of MM cell lines and primary MM cells, regardless of prior drug resistance, in a dose- and time-dependent manner}

To assess the efficacy of ART as an antimyeloma agent, we first evaluated its impact on the viability of the MM cells. ART was able to inhibit viability in clinically achievable concentrations [10] and in a time-dependent manner in IL-6-independent and -dependent MM cell lines (Suppl. Table S1A; Fig. 1A-E). The MM cell line RPMI $8226 / \mathrm{R} 5$ which is known to exhibit one of the highest BZ IC50s among MM cell lines [11] proved to be among the most sensitive to ART.

Motivated by this finding and by the clinical importance of drug resistance in treating MM, we sought to investigate the effects of ART in different models of
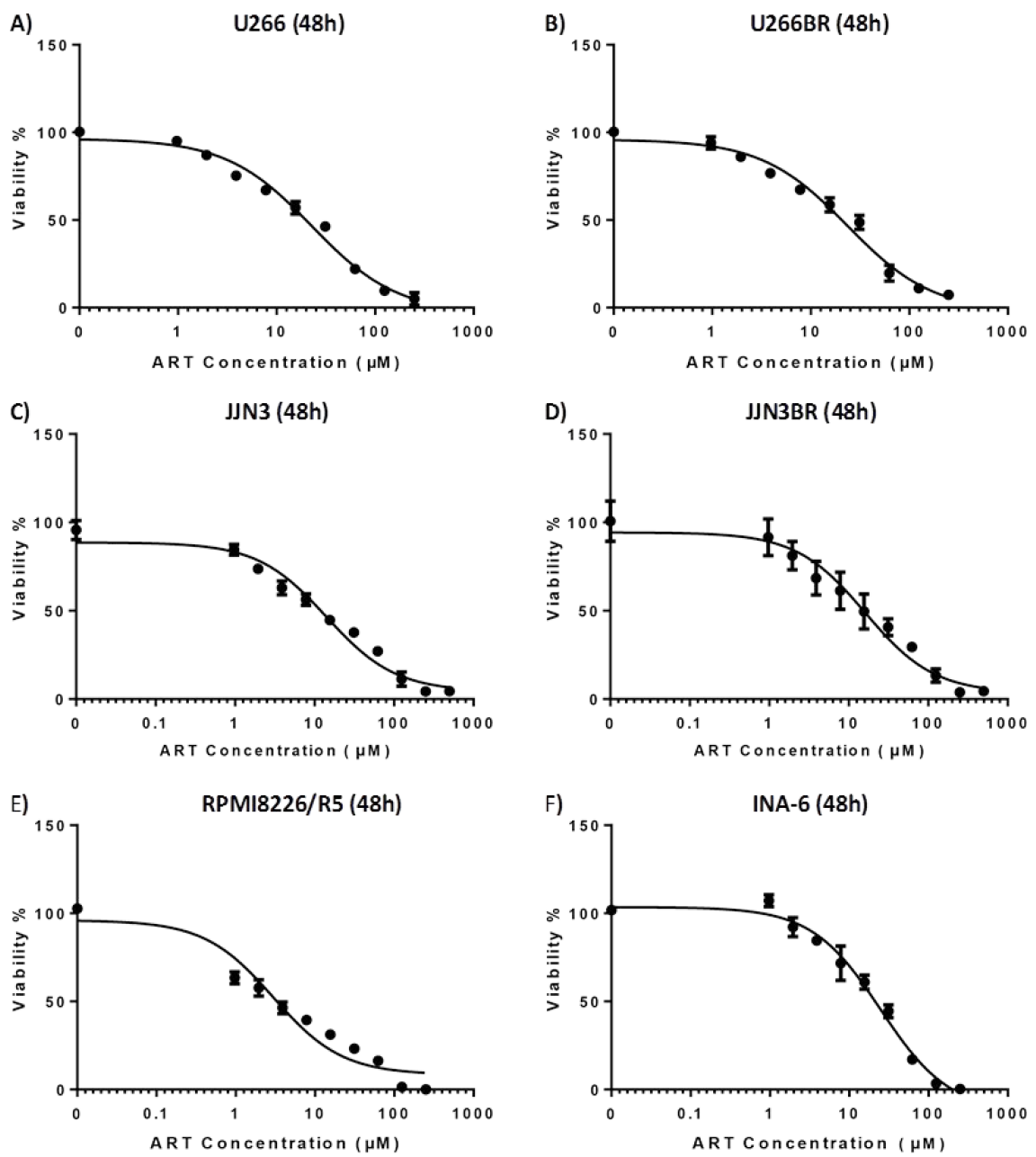

Figure 1: IC50 curves of various cell lines on 48 hour exposure to ART. A) U266 cell line B)U266BR cell line C) JJN3 cell line D) JJN3BR cell line E) RPMI8226/R5 F)INA-6 cell line
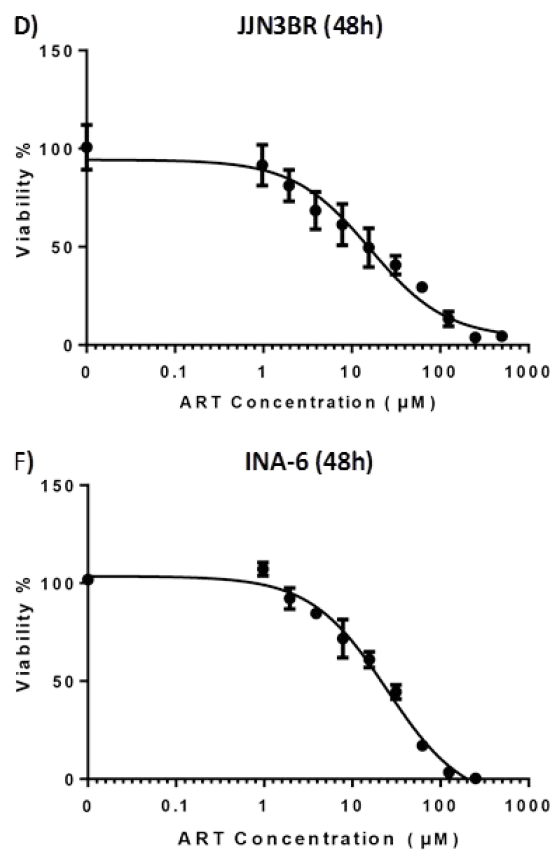

drug resistance. We developed two BZ-resistant (BR) sublines of BZ-sensitive cell lines JJN3 and U266 by exposing cells to increasing concentrations of BZ. JJN3BR and U266BR exhibited 20-fold increases in BZ IC50 (48 h), compared to BZ-naïve parental cells (Supplementary Fig. S1A and B). Treating JJN3BR and U266BR cells with ART resulted in no evidence of cross-resistance, compared to parental cell lines; similarly treating dexamethasone (DEX)-resistant cell line MM-1R and its DEX-sensitive mate MM.1S resulted also in no cross-resistance. In-vitro anti-MM effectiveness of ART was also evident in CD138selected primary MM cells from six patients with relapsed/ refractory MM (Supplementary Table S1B).

\section{ART induces non-caspase mediated apoptosis in MM cell lines}

We investigated the mechanism behind ART's ability to induce cell death in MM cells. In several cell lines, flow cytometry analyses of annexin $\mathrm{V}$ and PI positivity indicated that ART induced early and late apoptosis (Fig. 2A-E); BZ resistance had no effect on ART's ability to 
induce apoptosis. To further elucidate ART's mode of action, we determined the sequence and level of caspase activation. ART exposure initiated the extrinsic pathway of apoptosis with a brisk increase in levels of activated caspase- 8 at 3 h, followed by activation of caspase- 9 at 6 $\mathrm{h}$ (Fig. 3A). A prominent increase in the levels of effector caspase $3 / 7$ was evident after $12 \mathrm{~h}$ of ART exposure (Fig. $3 \mathrm{~A})$. To further examine the role of caspases in inducing apoptosis after ART exposure, we evaluated the impact of pan-caspase inhibitor Z-VAD-mfk on the effectiveness of ART. Z-VAD-mfk effectively inhibited caspase activation by ART (Fig. 3B) but did not block ART's effects on cell viability - at least $70 \%$ of ART's effect was retained in JJN3, U266, and RPMI 8226/R5 cells (Fig. 3C). Similar results were obtained in flow cytometry-based apoptosis assays (Supplementary Fig. S2A-B)
A)

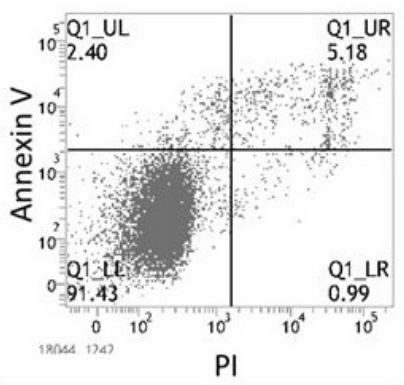

C)

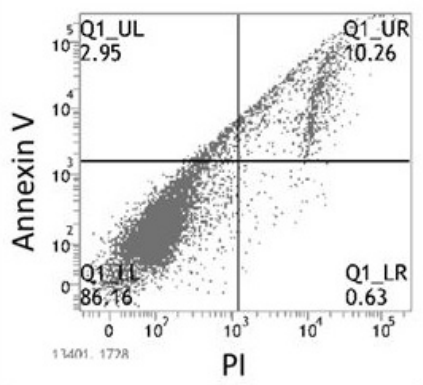

E)

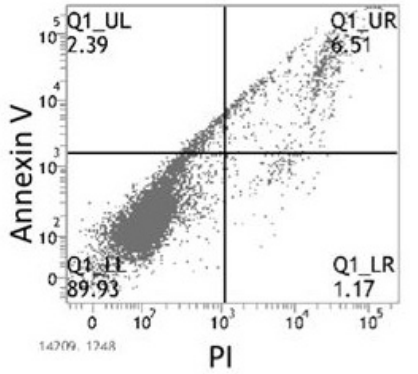

B)

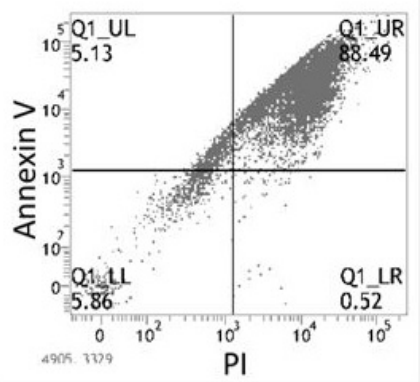

D)

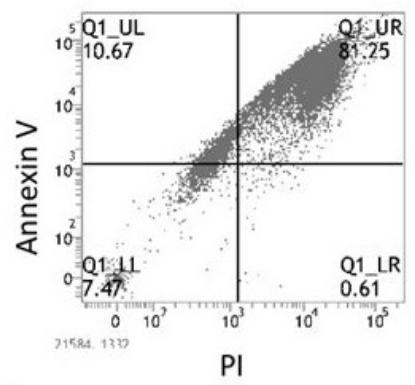

F)

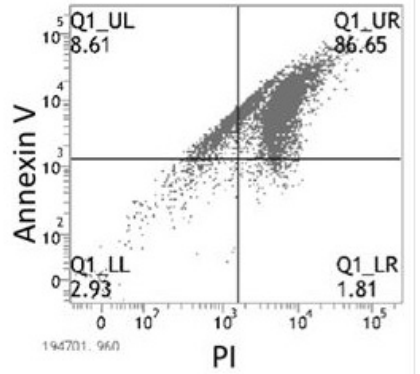

Figure 2: Flow cytometry assay for detection of early and late apoptosis through Annexin $\mathrm{V}$ and PI staining respectively. A) Untreated JJN3 cells (Control) B) JJN3 after 48 hour exposure to $125 \mu \mathrm{M}$ ART C) Untreated JJN3-BR cells (Control) D) JJN3-BR after 48 hour exposure to $125 \mu \mathrm{M}$ ART E) Untreated RPMI-8226 cells (Control) F) RPMI-8226 after 48 hour exposure to $125 \mu \mathrm{M}$ ART
ART-induced non-caspase mediated apoptosis is characterized by cytoplasmic and subsequent nuclear translocation of AIF and EndoG

To investigate the molecular effectors involved in ART's induction of non-caspase-mediated apoptosis, we examined the translocation of the two main mitochondrial factors implicated in this type of apoptosis, AIF and EndoG. When JJN3 cells were exposed to $125 \mu \mathrm{M}$ ART, AIF translocated from mitochondria to cytoplasm at $6 \mathrm{~h}$ and to the nucleus at $12 \mathrm{~h}$ (Fig. 4); the soluble apoptogenic AIF $\Delta 1-102 / 118$ isoform was readily detected in the
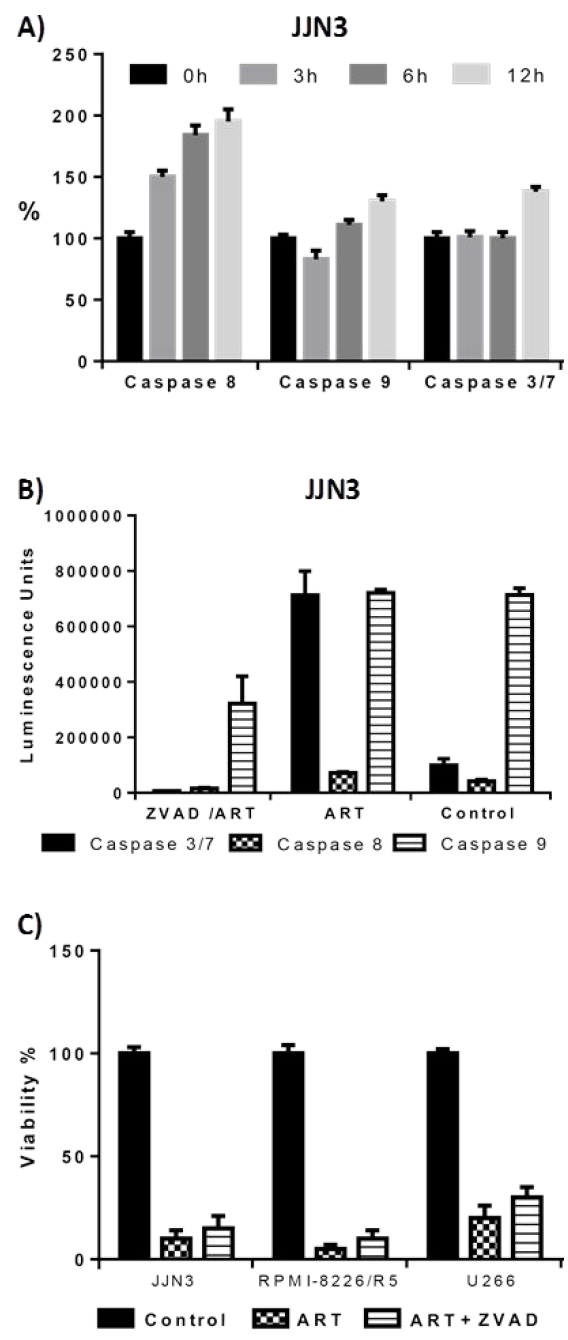

Figure 3: Quantification of activated caspases 3/7, 8, 9 through Caspase-Glo ${ }^{\circledR}$ assay (Figures A-B) and effect of caspase inhibition to ART's effect on cell viability (Figure C). A) Activated caspase 8,9, 3/7 levels compared with untreated cells ( 0 hour) after 3,6 and 12 hour exposure to 125 $\mu \mathrm{M}$ ART (JJN3 cells) Luminescence was normalized to that of untreated cells. B) Activated caspase levels (units are arbitrary luminescence units) after 48 hour exposure to $125 \mu \mathrm{M}$ ART and $200 \mu \mathrm{M}$ of the pan-caspase inhibitor Z-VADfmk. C) Effect of caspase inhibition to viability of the cells after 48 hour $125 \mu \mathrm{M}$ ART exposure. The effect of ART on cell viability is not affected by inhibition of caspases by Z-VADfmk. 
cytoplasmic fraction [12] (Fig. 4). Similarly, EndoG cytoplasmic and nuclear translocation were evident at $6 \mathrm{~h}$ and became more prominent at $12 \mathrm{~h}$, especially in the cytoplasmic fraction (Fig. 4). Because nuclear translocation of AIF and EndoG has been directly related to DNA damage and fragmentation [13], we evaluated the cleavage of PARP-1, a nuclear protein that repairs DNA damage [14] and whose cleavage is associated with apoptosis characterized by excessive DNA damage [15] . Indeed, cleavage of PARP-1 in the nuclear fraction was clearly evident at $24 \mathrm{~h}$, well after nuclear translocation of AIF and EndoG. Similar results were obtained for RPMI8226/R5 cells (Supplementary Fig. S3A and B).

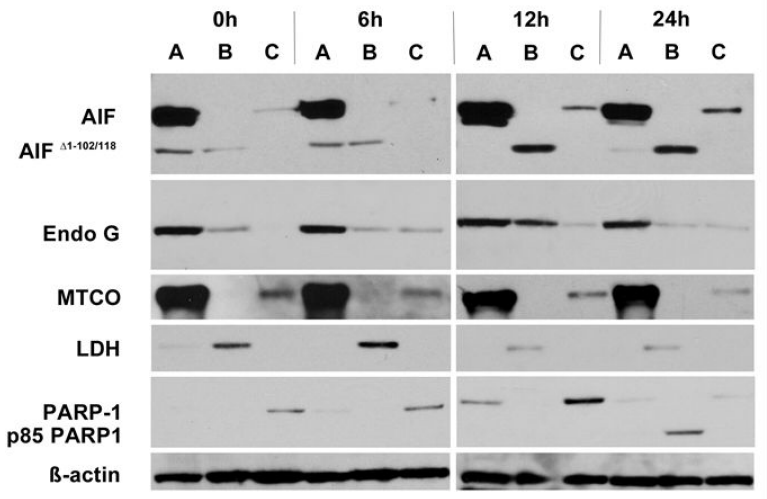

Figure 4: Treatment of JJN3 cells with ART $125 \mu M$ induces cytoplasmic and nuclear translocation of AIF and EndoG. Translocation of AIF and EndoG from the mitochondria to the cytoplasm is detected by Western Blot as early as $6 \mathrm{~h}$ after exposure to ART. MTCO is used as a mitochondrial marker, LDH is used as a cytoplasmic marker, PARP-1 is used as a nuclear marker and an indicator of apoptosis (cleavage from nucleus and presence of the $\mathrm{p} 85$ cleaved form of PARP-1). A: Mitochondrial fraction, B: Cytoplasmic fraction, C: Nuclear fraction
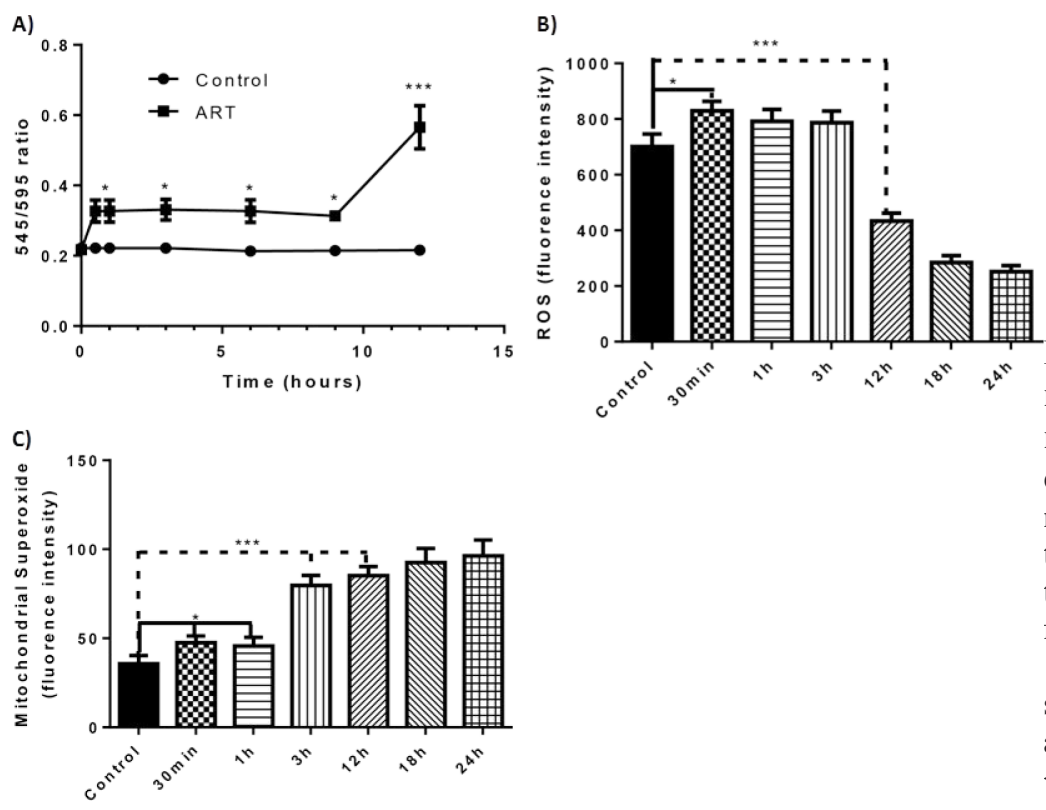

ART primarily targets mitochondria and induces abnormally low levels of ROS in MM cells

Because ART-mediated apoptosis occurs primarily through the non-caspase pathway, which evolves from mitochondrial factors, we examined the effects of ART on mitochondria by using JC-1 dye [16] to measure changes in mitochondrial membrane potential $(\Delta \Psi \mathrm{m})$ in JJN3 and RPMI-8226 cells. The ratio of the 545 -nm (green fluorescence) and 595-nm (red fluorescence) emissions readings was used as a surrogate for the $\Delta \Psi \mathrm{m}$, since this ratio is not affected by factors such as mitochondrial size, shape, and density, which may influence singlecomponent fluorescence signals. As early as 30 minutes after exposure to ART, a small but statistically significant change occurred in the $\Delta \Psi \mathrm{m}$. This change persisted for 12 hours, when a brisk and continuous increase in the $\Delta \Psi \mathrm{m}$ was observed (Fig. 5A; Supplementary Fig. S4A).

Because mitochondrial function plays a pivotal role in the cellular ROS balance [17], we examined the effects of ART treatment on the ROS status and production of mitochondria-derived superoxide. ART treatment resulted in a small but steep increase in ROS level within 30 minutes after drug exposure; however, by $12 \mathrm{~h}$, the ROS level was well below that of untreated control cells (Fig. 5B). The decrease in ROS level cannot be due to a reduced number of viable cells because annexin $\mathrm{V}$ staining (indicating apoptotic cells) was negligible at the 12-h time point (Supplementary Fig. S4B). Interestingly, the reduced cellular ROS level was accompanied by high levels of mitochondria-derived superoxide (Fig. 5C). The paradoxical reduction in cellular ROS levels and increase in mitochondrial superoxide production that occurred $12 \mathrm{~h}$ after ART treatment also occurred in RPMI-8226/R5 MM cells (Supplementary Fig. S4D-E). In contrast, $12 \mathrm{~h}$ after treating cells with $\mathrm{BZ}$ at a comparable effective dosage
Figure 5: Effect of ART to the mitochondrial membrane potential, cellular level of ROS and mitochondrial superoxide through the course of time and timing of apoptosis. A) Change of the mitochondrial membrane potential $(\Delta \Psi \mathrm{m})$ as portrayed through the change of the $545 \mathrm{~nm} / 595 \mathrm{~nm}$ ratio in relation to time (JJN3 cells). B) ROS levels (units are arbitrary fluorescence units) at different time intervals of ART $125 \mu \mathrm{M}$ exposure (JJN3 cells) C) Mitochondrial derived superoxide levels (units are arbitrary fluorescence units) at different time intervals of ART $125 \mu \mathrm{M}$ exposure. ${ }^{*} P$ $<0.05$ and $P>0.01, * * * P<0.001$ 
(10nM) the ROS level was clearly higher than that of control cells (Supplementary Fig. S4C).

\section{ART's efficacy depends on the levels of intracellular bivalent iron}

An iron dependent mechanism that directly affects the redox cycle of the parasite has been proposed for the antimalarial action of ART [18]. We therefore proceeded to examine the effect of iron in ART's anti-MM efficacy. Supplementation of growth medium with bivalent iron in the form of Iron (II) sulfate heptahydrate at a concentration of $0.8 \mathrm{mg} / \mathrm{L}$ (i.e. within the normal range of iron levels in human blood $(0.6-1.7 \mathrm{mg} / \mathrm{L})$ ) drastically increased ART's anti-MM efficacy, as evidenced by a marked decrease in its IC50 in JJN3, INA-6, RPMI-8286, and U266 cell lines (Supplemental Table S2). This decrease in IC50 was much less pronounced when the protein-bound form of iron, holotransferrin, was used and did not achieve statistical significance when the trivalent form of iron (Iron(III) citrate) was used at the same concentration, as was the bivalent form (Supplemental Table S2). Deferoxamine, an iron chelator that can enter the cells [19], exerted clear antagonism to ART (Supplementary Fig. S5A). The potentiating effect of bivalent iron and the inhibitory effects of deferoxamine were also reflected in levels of ROS and mitochondrial superoxide at the various time points (Supplementary Fig. S5B and C). To clarify whether ART's anti-MM efficacy is potentiated by intracellular or extracellular bivalent iron, we examined the combined effects of ART and EDTA, a potent metal chelator that does not enter the cells [20]. Addition of EDTA had no statistically significant effect on ART's IC50 (Supplementary Fig. S5D), suggesting that it is the intracellular bivalent form of iron which potentiates ART's efficacy.

\section{ART exhibits synergy with various anti-MM agents}

Due to the different pathway of inducing apoptosis in ART we then tried to examine the effect of the combination of ART with the known anti-MM agents, DEX, doxorubicin(DOX) and BZ that are known to induce apoptosis through the caspase dependent pathway with a high ROS accumulation. While ART induces apoptosis primarily through a non-caspase dependent pathway, anti-MM agents DEX, doxorubicin(DOX), and BZ induce apoptosis through the caspase dependent pathway characterized by high levels of ROS [21-23]. For this purpose the "Synergy" program was applied as described in the methods section and the Interaction Index (II) was used as an index of interaction evaluation. Values greater than 1 are consistent with antagonistic effect, values equal to 1 are consistent with additive effect and values less than
1 are consistent with a synergistic effect. ART exhibited profound synergism with all tested agents in medium to low dosages. At high dosages the effect was either additive (DEX, BZ) or slightly antagonistic (DOX) (Figure 6A-C). However it should be noted that in the high concentrations the IC50 values were exceeded for all agents.

\section{DISCUSSION}

Artemisinin and its derivative ART have been reported to have anticancer activity in many different in vitro tumor models [6]. This report establishes ART's in vitro effectiveness against $\mathrm{MM}$ cells and elucidates the mechanisms by which this agent induces apoptosis of MM cells. Our observations suggest that ART induces apoptosis primarily through the non-caspase mediated pathway and that the mitochondria are ART's primary target in MM cells. Furthermore, initiation of apoptosis was characterized by low levels of cellular ROS levels and a marked increase in mitochondrial superoxide, an effect that has not been previously reported for other drugs.

Non-caspase mediated apoptosis has not been studied extensively [24]. The main factors implicated in this form of apoptosis are the AIF and EndoG [25]. In our experiments ART induced non-caspase apoptosis was related to translocation of AIF and EndoG initially from the mitochondria to the cytoplasm and subsequently to the nucleus. While whether or not non-caspase mediated apoptosis is the dominant pathway of ART induced apoptosis seems to be cell type dependent [7], Zhou et al [26] also found non-caspase apoptosis in lung adenocarcinoma cells as the main mechanism of ART induced cell death. Furthermore, ART caused the release of the apoptogenic isoform $\Delta 1-102 / 118$ of AIF from the mitochondria, which has been shown to be an aftermath of the outer mitochondrial membrane permeabilization (OMMP) [12]. The OMMP leads to a loss to the $\Delta \Psi \mathrm{m}$ and an increase in ROS [27]. It should be noted that these two events coincided early in the timeline of ART exposure and were concurrent with the release of both $\Delta 1-102 / 118$ AIF and EndoG to the cytoplasm. The nuclear translocation of AIF and EndoG initiated apoptosis in MM cells a finding consistent with the effects of these two factors in other type of cells [28, 29]. Moreover this nuclear translocation preceded the cleavage of PARP-1, in accordance with other reports [30], providing further evidence that the cell death of MM cells from ART exposure is through programmed apoptosis. PARP-1 cleavage, an indicator of late apoptosis [31], followed the nuclear translocation of AIF and EndoG supporting thus the role of these two factors in the ART induced apoptosis in MM cells.

While the biggest difference in $\Delta \Psi \mathrm{m}$ was found at 12 hours, changes that were statistically significant were found as early as 30 minutes after drug exposure. This was accompanied by a small but brisk increase in the ROS level of the cells. It should be noted that while 
the mitochondria are the primary target of ART, for the caspase dependent pathway of apoptosis it is the extrinsic pathway that is activated first. This activation is an early event and occurs at the time of increased ROS production. It has been shown that increased ROS levels can activate the extrinsic pathway of caspase dependent apoptosis by ROS induced sphingomyelinase activation, ceramide production and death receptor clustering [32]. However, at least in MM cell lines, it does not seem to be the increased level of ROS that actually induces apoptosis but the way ART interferes with the redox status of the cell.

The increased mitochondrial superoxide production from the beginning of ART exposure does not translate to an overall increase in cellular ROS levels. In fact the opposite happens; at 12 hours when the mitochondrial derived superoxide level is at its highest, cellular ROS level is well below that of the untreated control. This paradox could imply that ART directly interferes with the Fenton reaction for production of free radicals from superoxide. In this reaction, bivalent iron plays a fundamental role, and inhibition of iron reduction from the bivalent to the trivalent form diminishes production

A)

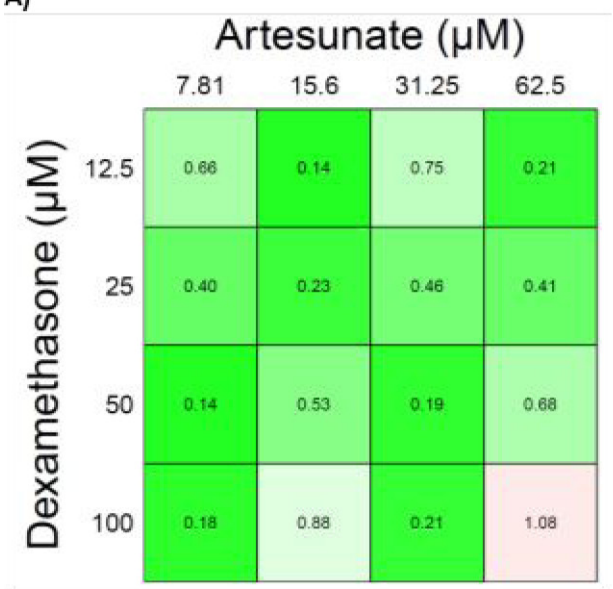

c)

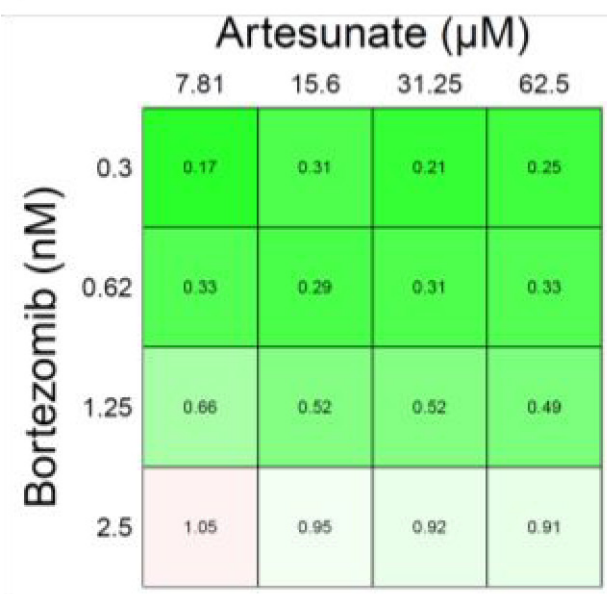

of hydroxyl and free oxygen radicals [33]. This proposed mechanism is supported by our finding that ART's effects on mitochondria and induction of apoptosis are strongly related to intracellular levels of bivalent iron. The interaction of ART with bivalent iron in malarial parasites also has been implicated in the drug's anti-plasmodial effect [34].

Early and sustained mitochondrial superoxide overproduction combined with the early change in the $\Delta \Psi \mathrm{m}$, as observed in our experiments, is consistent with a complex I mitochondrial dysfunction [17]. Interestingly, complex I function is highly dependent on the FMN cofactor, which passes electrons through a chain of seven FeS (iron sulfur) centers to the CoQ reduction site [35], where the bivalent or trivalent state of iron is of crucial importance for the electron transport. Non-caspase mediated apoptosis has been linked with loss of cellular respiration and dysfunction of mitochondrial complex I that precedes nuclear translocation of AIF and EndoG [36]; this is consistent with our findings from MM cell lines treated with ART. In addition, it has been shown that, even in caspase-dependent $\Delta \Psi \mathrm{m}$ dissociation, the

B)
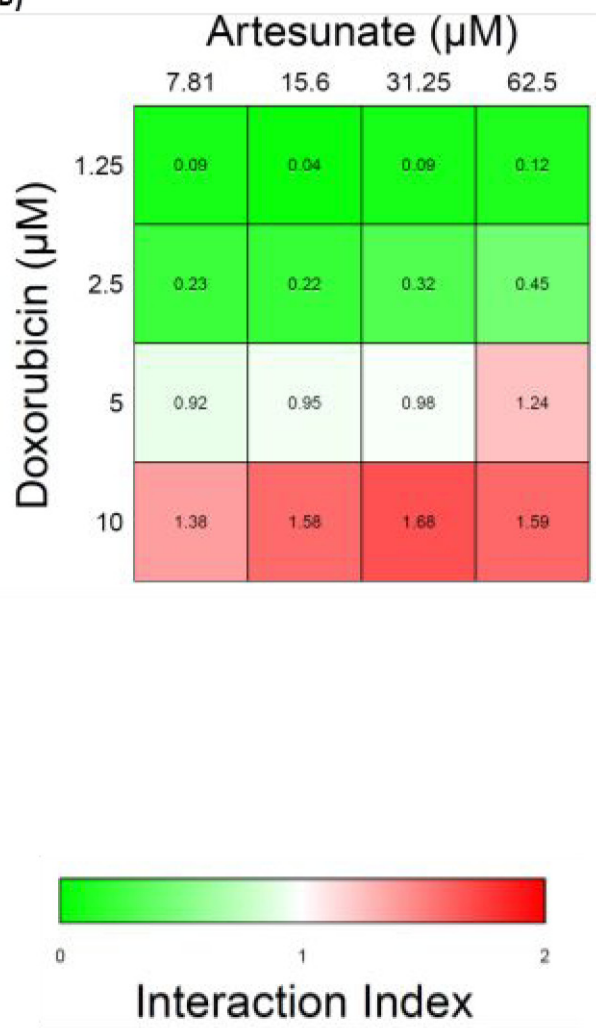

Figure 6: Heatmaps of the interaction index of Artesunate with various frontline anti-MM agents. A) Artesunate and Dexamethasone, B) Artesunate and Doxorubicin, C) Artesunate and Bortezomib. Values above 1 (red) are consistent with an antagonistic effect, values equal to 1 (white) are consistent with additive effect and values less than 1 (green) are consistent with a synergistic effect. 
iron-containing NADH-ubiquinone oxireductase subunit of complex I is primarily affected [37]. Dysfunction of Complex I has been linked with superoxide overproduction along with $\Delta \Psi \mathrm{m}$ alterations [38]. Similar findings pertain to the iron-containing subunits of mitochondrial complexes II [39] and III [40]. Also of importance is the fact that heme seems to be one of ART's target molecules in cancer cells, where it reduces heme (bivalent iron) to hemin (trivalent iron) [41]. Heme is essential to many protein complexes and one of them, cytochrome $\mathrm{C}$, plays a fundamental role in the electron transport chain of the mitochondria [42]. Thus the mitochondrion, with its abundance of iron provides an ideal environment for the action of ART. Although we have not shown depletion of bivalent iron as a direct effect of ART, circumstantial evidence - as laid out in this study - suggest $\mathrm{Fe}^{+2}$ as a target of ART. We have summarized this in Supplementary Fig. S6. In clinical studies for malaria ART was shown to have an excellent toxicity profile $[9,43]$, with the most significant finding a transient minimal reduction of the reticulocyte count in a phase I dose escalation study [10]. In vitro studies with human peripheral blood mononuclear cells found the IC50 to be more than $100 \mu \mathrm{M}$ a dosage which as shown here exceeds several times the IC50 in MM cells [44]. When dihydroartemisinin (DHA) - the primary metabolite of ART- was tested in cultures of human CD34+ selected cells only a transient effect on erythroid maturation was evident. No differences in cell number between cells cultured with and without DHA were seen in long term cultures, regardless of the time exposure to DHA [45]. ART was able to overcome resistance in both $\mathrm{BZ}$ as well as DEX resistant MM cell lines regardless of whether the resistance was native or acquired. Additionally, we were able to show synergy between ART and several frontline anti-MM agents, which are known to cause caspase-dependent, high-ROS apoptosis. Through these unique mechanisms ART not only has the ability to act independently but can also complement and augment the effect of other anti-myeloma agents. Primary BZ resistance of a subpopulation of MM cells in the clinical setting has been identified as the main mechanism failure of BZ to eradicate the disease [46]. These data provide the rationale for further "in vivo" experiments to establish the therapeutic potential of ART either alone or in combination with other agents especially in the relapsed/refractory MM setting.

In conclusion ART is an effective single agent, has synergy with known myeloma agents and can overcome resistance to $\mathrm{BZ}$ in preclinical models of $\mathrm{MM}$. One possible explanation for these beneficial effects is that ART induces apoptosis through a unique mechanism of non-caspase mediated apoptosis and low levels of cellular ROS. While more work needs to be done to elucidate the entire mechanism of the ART-induced initial ROS production, mitochondrial dysfunction and the specific redox signaling sequelae that result in apoptosis, and for the establishment of ART's efficacy in MM in vivo, ART could potentially be the forerunner of a whole new class of agents for the treatment of relapsed/refractory MM.

\section{MATERIALS AND METHODS}

\section{Cell models, cell culture, and experimental conditions}

MM cell lines RPMI 8226/R5, INA-6, MM-1S, MM-1R, KMS-11, ARH-77, JJN3, U266; BZ-resistant (BR) sublines JJN3BR and U266BR; and patientderived primary MM cells were cultured in RPMI-1640 (Corning) supplemented with 10\% heat-inactivated fetal bovine serum (Atlas Biologicals), $100 \mathrm{U} / \mathrm{ml}$ penicillin/ streptomycin, and 2-mM L-glutamine and were incubated at $37^{\circ} \mathrm{C}$ with $5 \% \mathrm{CO}_{2}$. Interleukin (IL)-6-dependent cells and purified plasma cells from patients were supplemented with 2-ng/ml IL-6 (R\&D Systems). All cell lines were authenticated by a method of copy number variant (CNV) fingerprinting developed by Jonathan Keats and P. Leif Bergsagel [47].

Patient samples were collected under a protocol approved by the Institutional Review Board of the University of Arkansas for Medical Sciences (UAMS). Informed consent was obtained in accordance with the Declaration of Helsinki. Mononuclear cells from bone marrow aspirates were isolated by density gradient centrifugation over Ficoll-Hipaque Plus. Plasma cells were then isolated with immunomagnetic bead selection for CD138+ cells in a Midi MACS LS column following the manufacturer's recommendations (Miltenyi Biotec). Plasma cell purity was confirmed by flow cytometric analysis with phycoreythrin-conjugated CD38 and CD45 antibodies (Miltenyi Biotec).

Stock solutions of ART (Sigma-Aldrich) and BZ (LC Laboratories) were prepared in dimethyl sulfoxide (DMSO) and used as indicated, with a final vehicle concentration that did not exceed $0.5 \%$ (vol/vol). All chemicals, unless otherwise indicated, were purchased from Fisher Scientific.

\section{Cell extract preparation and Western blot analysis}

Mitochondrial, cytoplasmic, and nuclear fractions from cultured cell lines JJN3 and RPMI-8226/R5 were isolated with Cell Fractionation Kit Standard from (Mito sciences). Protein samples were fractionated with SDS PAGE and transferred onto PVDF membrane. Antibodies for apoptosis inducing factor (AIF), poly (ADP ribose) polymerase-1 (PARP-1), lactate dehydrogenase (LDH), and mitochondrial cytochrome $\mathrm{C}$ oxidase (MTCO) were purchased from Abcam, antibody for $\beta$-actin was purchased from Cell Signalling Technology, and the 
antibody for endonuclease $\mathrm{G}$ (EndoG) was purchased from EMD Millipore.

\section{Cell viability assays}

Cell viability was measured with CellTiter 96 AQueous Non-Radioactive Cell Proliferation Assay (MTS) (Promega) as published elsewhere [48]. Briefly, myeloma cells were seeded in 96 wells at a concentration of approximately $2 \times 10^{4}$ cells per well. After incubating in culture medium alone for 24 hours, anti-myeloma agents were added in, in serial 2-fold dilutions titrated according to the potency of each agent. MTS was added to each well at the time points indicated for each experiment. Absorbance was read at a $490 \mathrm{~nm}$ wavelength according to the manufacturers recommendations. All tests were done in triplicates.

\section{Statistical analysis}

IC50 calculations and the respective curves were generated by GraphPad Prism v6.0 Software. The effect of the interaction of different agents on combination treatment (synergistic, additive, antagonistic) was analyzed with the freeware program "Synergy" based on the R statistical language ( $\mathrm{R}$ v2.13.1) as published elsewhere [49]. For each interaction-effect experiment, the ChouTalalay method [50] was primarily used and results were verified with the Plummer method [51]. For comparisons of treatment groups, unpaired $t$-tests (Mann-Whitney), paired $t$-tests, and one-way or two-way ANOVA (where appropriate) were performed. For ANOVA, Bonferroni posthoc analysis was used to compare treatment groups. All analyses were performed with GraphPad Prism v6.0. Differences with a $\mathrm{P}$ value $\leq 0.05$ were considered significant.

\section{Apoptosis assays}

Staining cells with annexin $\mathrm{V}$ and propidium iodide (PI) for the detection of early and late apoptosis, respectively, was performed according to the manufacturer's specifications (Beckton Dickinson) and as published elsewhere [52]. Data were collected using the FACSuite software on a FACSVerse flow cytometer (Beckton Dickinson). A minimum of 10,000 events were analyzed for each experiment. All experiments were conducted in triplicate.

\section{Mitochondrial membrane potential $(\Delta \Psi \mathrm{m})$}

The change in the $\Delta \Psi \mathrm{m}$ was detected by staining with JC-1 dye (R\&D Systems) as described elsewhere [53]. Briefly, after exposure to the indicated drug for the indicated time period, cells were incubated with $3.5 \mu \mathrm{M}$ $\mathrm{JC}-1$ for 15 minutes at $37^{\circ} \mathrm{C}$. After this incubation period cells were then rinsed and transferred to a 96-well plate for fluorescence measurement by a plate reader with the following settings: excitation $485 \mathrm{~nm}$, emission $545 \mathrm{~nm}$ and $595 \mathrm{~nm}$, cutoff $530 \mathrm{~nm}$. The ratio of measurements at $545 \mathrm{~nm} / 595 \mathrm{~nm}$ was used as the measurement of the $\Delta \Psi \mathrm{m}$.

\section{ROS and mitochondria-derived superoxide detection and quantification}

For the determination of ROS and mitochondrial derived superoxide generation, cells were treated with drugs at indicated doses and time. On the day of the analysis, $0.5 \times 10^{6}$ cells were resuspended in $1 \mathrm{~mL}$ prewarmed PBS and incubated with $10 \mu \mathrm{M} \mathrm{H}_{2}$ DCFDA (Life Technologies) or in $0.5 \mathrm{ml}$ DPBS with $5 \mu \mathrm{M}$ MitoSOX (Life Technologies) for $30 \mathrm{~min}$ at $37^{\circ} \mathrm{C}$. Then, cells were washed with ice-cold PBS and subjected to flow cytometry using the FACSVerse (Becton Dickinson). Data were analyzed with FC Express software (De Novo).

\section{Caspase activation and quantification assay}

Identification and quantification of the activated caspases $3 / 7,8$, and 9 was performed through the use of the CaspaseGlo assay (Promega), as published elsewhere [54]. Pancaspase inhibitor Z-VAD-fmk was purchased from R\&D and was applied at a concentration of $200 \mu \mathrm{M}$ every 24 hours unless otherwise indicated.

\section{Authors' Contributions:}

Conception and design of the study: XP, $\mathrm{CH}$.

Development of methodology: XP, CH, SJ, TG, ET, QZ, CS, RT

Acquisition of data: XP, SJ, TG, QZ, RT

Analysis and interpretation of data: XP, CH, SJ, JE,

BB

Writing, review, and/or revision of the manuscript: $\mathrm{XP}, \mathrm{CH}, \mathrm{BB}, \mathrm{JE}$

Administrative, technical and/or material support: $\mathrm{CH}, \mathrm{BB}, \mathrm{JE}$

Study supervision: $\mathrm{CH}$

\section{ACKNOWLEDGMENTS}

This work was supported by the National Cancer Institute, Bethesda, MD (CA 55813). We would like also to thank Margaret E. Brenner for editorial assistance. 


\section{Conflicts of Interest:}

B.B. has received research funding from Celgene and Millennium and is a consultant to Celgene, Millennium, Onyx, and Amgen. He is a co-inventor on patents and patent applications related to use of gene expression profiling in cancer medicine that have been licensed to Myeloma Health, LLC, but he has no financial interest in the company. All other authors have no conflicts of interest to declare.

\section{REFERENCES}

1. Kyle RA. Long-term survival in multiple myeloma. The New England journal of medicine. 1983; 308(6):314-316.

2. Nair B, van Rhee F, Shaughnessy JD, Jr., Anaissie E, Szymonifka J, Hoering A, Alsayed Y, Waheed S, Crowley J and Barlogie B. Superior results of Total Therapy 3 (200333 ) in gene expression profiling-defined low-risk multiple myeloma confirmed in subsequent trial 2006-66 with VRD maintenance. Blood. 2010; 115(21):4168-4173.

3. Zhan F, Barlogie B, Mulligan G, Shaughnessy JD, Jr. and Bryant B. High-risk myeloma: a gene expression based riskstratification model for newly diagnosed multiple myeloma treated with high-dose therapy is predictive of outcome in relapsed disease treated with single-agent bortezomib or high-dose dexamethasone. Blood. 2008; 111(2):968-969.

4. Kumar SK, Lee JH, Lahuerta JJ, Morgan G, Richardson PG, Crowley J, Haessler J, Feather J, Hoering A, Moreau P, LeLeu X, Hulin C, Klein SK, Sonneveld P, Siegel $\mathrm{D}$, Blade $\mathrm{J}$, et al. Risk of progression and survival in multiple myeloma relapsing after therapy with IMiDs and bortezomib: a multicenter international myeloma working group study. Leukemia : official journal of the Leukemia Society of America, Leukemia Research Fund, UK. 2012; 26(1):149-157.

5. Chen H, Sun B, Pan S, Jiang $H$ and Sun $X$. Dihydroartemisinin inhibits growth of pancreatic cancer cells in vitro and in vivo. Anti-cancer drugs. 2009; 20(2):131-140.

6. Mercer AE, Maggs JL, Sun XM, Cohen GM, Chadwick J, O'Neill PM and Park BK. Evidence for the involvement of carbon-centered radicals in the induction of apoptotic cell death by artemisinin compounds. The Journal of biological chemistry. 2007; 282(13):9372-9382.

7. Cabello CM, Lamore SD, Bair WB, 3rd, Qiao S, Azimian $\mathrm{S}$, Lesson JL and Wondrak GT. The redox antimalarial dihydroartemisinin targets human metastatic melanoma cells but not primary melanocytes with induction of NOXAdependent apoptosis. Investigational new drugs. 2012; 30(4):1289-1301.

8. Kast RE, Boockvar JA, Bruning A, Cappello F, Chang WW, Cvek B, Dou QP, Duenas-Gonzalez A, Efferth T, Focosi D, Ghaffari SH, Karpel-Massler G, Ketola K, Khoshnevisan
A, Keizman D, Magne N, et al. A conceptually new treatment approach for relapsed glioblastoma: coordinated undermining of survival paths with nine repurposed drugs (CUSP9) by the International Initiative for Accelerated Improvement of Glioblastoma Care. Oncotarget. 2013; 4(4):502-530.

9. Rosenthal PJ. Artesunate for the treatment of severe falciparum malaria. The New England journal of medicine. 2008; 358(17):1829-1836.

10. Miller RS, Li Q, Cantilena LR, Leary KJ, Saviolakis GA, Melendez V, Smith B and Weina PJ. Pharmacokinetic profiles of artesunate following multiple intravenous doses of 2,4 , and $8 \mathrm{mg} / \mathrm{kg}$ in healthy volunteers: phase $1 \mathrm{~b}$ study. Malaria journal. 2012; 11:255.

11. Buzzeo R, Enkemann S, Nimmanapalli R, Alsina M, Lichtenheld MG, Dalton WS and Beaupre DM. Characterization of a R115777-resistant human multiple myeloma cell line with cross-resistance to PS-341. Clinical cancer research : an official journal of the American Association for Cancer Research. 2005; 11(16):6057-6064.

12. Churbanova IY and Sevrioukova IF. Redox-dependent changes in molecular properties of mitochondrial apoptosisinducing factor. The Journal of biological chemistry. 2008; 283(9):5622-5631.

13. Strauss G, Westhoff MA, Fischer-Posovszky P, Fulda S, Schanbacher M, Eckhoff SM, Stahnke K, Vahsen N, Kroemer G and Debatin KM. 4-hydroperoxycyclophosphamide mediates caspase-independent T-cell apoptosis involving oxidative stress-induced nuclear relocation of mitochondrial apoptogenic factors AIF and EndoG. Cell death and differentiation. 2008; 15(2):332-343.

14. Dantzer F, de La Rubia G, Menissier-De Murcia J, Hostomsky Z, de Murcia G and Schreiber V. Base excision repair is impaired in mammalian cells lacking Poly(ADPribose) polymerase-1. Biochemistry. 2000; 39(25):75597569.

15. Gilliams-Francis KL, Quaye AA and Naegele JR. PARP cleavage, DNA fragmentation, and pyknosis during excitotoxin-induced neuronal death. Experimental neurology. 2003; 184(1):359-372.

16. Salvioli S, Ardizzoni A, Franceschi $\mathrm{C}$ and Cossarizza A. JC-1, but not DiOC6(3) or rhodamine 123, is a reliable fluorescent probe to assess delta psi changes in intact cells: implications for studies on mitochondrial functionality during apoptosis. FEBS letters. 1997; 411(1):77-82.

17. Murphy MP. How mitochondria produce reactive oxygen species. The Biochemical journal. 2009; 417(1):1-13.

18. Posner GH, McGarvey DJ, Oh CH, Kumar N, Meshnick SR and Asawamahasadka W. Structure-activity relationships of lactone ring-opened analogs of the antimalarial 1,2,4-trioxane artemisinin. Journal of medicinal chemistry. 1995; 38(4):607-612.

19. Halliwell B. Protection against tissue damage in vivo by desferrioxamine: what is its mechanism of action? Free 
radical biology \& medicine. 1989; 7(6):645-651.

20. Harding DP and Poole-Wilson PA. Calcium exchange in rabbit myocardium during and after hypoxia: effect of temperature and substrate. Cardiovascular research. 1980; 14(8):435-445.

21. Luanpitpong S, Chanvorachote P, Nimmannit U, Leonard SS, Stehlik C, Wang L and Rojanasakul Y. Mitochondrial superoxide mediates doxorubicin-induced keratinocyte apoptosis through oxidative modification of ERK and Bcl-2 ubiquitination. Biochemical pharmacology. 2012; 83(12):1643-1654.

22. Bera S, Greiner S, Choudhury A, Dispenzieri A, Spitz DR, Russell SJ and Goel A. Dexamethasone-induced oxidative stress enhances myeloma cell radiosensitization while sparing normal bone marrow hematopoiesis. Neoplasia. 2010; 12(12):980-992.

23. Perez-Galan P, Roue G, Villamor N, Montserrat E, Campo $\mathrm{E}$ and Colomer D. The proteasome inhibitor bortezomib induces apoptosis in mantle-cell lymphoma through generation of ROS and Noxa activation independent of p53 status. Blood. 2006; 107(1):257-264.

24. Elmore S. Apoptosis: a review of programmed cell death. Toxicologic pathology. 2007; 35(4):495-516.

25. Lorenzo HK and Susin SA. Mitochondrial effectors in caspase-independent cell death. FEBS letters. 2004; 557(13):14-20.

26. Zhou C, Pan W, Wang XP and Chen TS. Artesunate induces apoptosis via a Bak-mediated caspase-independent intrinsic pathway in human lung adenocarcinoma cells. Journal of cellular physiology. 2012; 227(12):3778-3786.

27. Tait SW and Green DR. Mitochondria and cell death: outer membrane permeabilization and beyond. Nature reviews Molecular cell biology. 2010; 11(9):621-632.

28. Loeffler M, Daugas E, Susin SA, Zamzami N, Metivier D, Nieminen AL, Brothers G, Penninger JM and Kroemer G. Dominant cell death induction by extramitochondrially targeted apoptosis-inducing factor. FASEB journal : official publication of the Federation of American Societies for Experimental Biology. 2001; 15(3):758-767.

29. Susin SA, Daugas E, Ravagnan L, Samejima K, Zamzami N, Loeffler M, Costantini P, Ferri KF, Irinopoulou T, Prevost MC, Brothers G, Mak TW, Penninger J, Earnshaw WC and Kroemer G. Two distinct pathways leading to nuclear apoptosis. The Journal of experimental medicine. 2000; 192(4):571-580.

30. Soldani C and Scovassi AI. Poly(ADP-ribose) polymerase-1 cleavage during apoptosis: an update. Apoptosis : an international journal on programmed cell death. 2002; 7(4):321-328.

31. Lee TJ, Kim EJ, Kim S, Jung EM, Park JW, Jeong SH, Park SE, Yoo YH and Kwon TK. Caspase-dependent and caspase-independent apoptosis induced by evodiamine in human leukemic U937 cells. Molecular cancer therapeutics. 2006; 5(9):2398-2407.
32. Zhang AY, Yi F, Jin S, Xia M, Chen QZ, Gulbins E and Li PL. Acid sphingomyelinase and its redox amplification in formation of lipid raft redox signaling platforms in endothelial cells. Antioxidants \& redox signaling. 2007; 9(7):817-828.

33. Welch KD, Davis TZ and Aust SD. Iron autoxidation and free radical generation: effects of buffers, ligands, and chelators. Archives of biochemistry and biophysics. 2002; 397(2):360-369.

34. O'Neill PM, Barton VE and Ward SA. The molecular mechanism of action of artemisinin--the debate continues. Molecules. 2010; 15(3):1705-1721.

35. Sazanov LA. Respiratory complex I: mechanistic and structural insights provided by the crystal structure of the hydrophilic domain. Biochemistry. 2007; 46(9):2275-2288.

36. Lartigue L, Kushnareva Y, Seong Y, Lin H, Faustin B and Newmeyer DD. Caspase-independent mitochondrial cell death results from loss of respiration, not cytotoxic protein release. Molecular biology of the cell. 2009; 20(23):48714884.

37. Ricci JE, Munoz-Pinedo C, Fitzgerald P, Bailly-Maitre B, Perkins GA, Yadava N, Scheffler IE, Ellisman MH and Green DR. Disruption of mitochondrial function during apoptosis is mediated by caspase cleavage of the p75 subunit of complex I of the electron transport chain. Cell. 2004; 117(6):773-786.

38. Korshunov SS, Skulachev VP and Starkov AA. High protonic potential actuates a mechanism of production of reactive oxygen species in mitochondria. FEBS letters. 1997; 416(1):15-18.

39. Ishii T, Miyazawa M, Onouchi H, Yasuda K, Hartman PS and Ishii N. Model animals for the study of oxidative stress from complex II. Biochimica et biophysica acta. 2013; 1827(5):588-597.

40. Turrens JF, Alexandre A and Lehninger AL. Ubisemiquinone is the electron donor for superoxide formation by complex III of heart mitochondria. Archives of biochemistry and biophysics. 1985; 237(2):408-414.

41. Zhang S and Gerhard GS. Heme mediates cytotoxicity from artemisinin and serves as a general anti-proliferation target. PloS one. 2009; 4(10):e7472.

42. Wiedemann FR, Vielhaber S, Schroder R, Elger CE and Kunz WS. Evaluation of methods for the determination of mitochondrial respiratory chain enzyme activities in human skeletal muscle samples. Analytical biochemistry. 2000; 279(1):55-60.

43. McGready R, Lee SJ, Wiladphaingern J, Ashley EA, Rijken MJ, Boel M, Simpson JA, Paw MK, Pimanpanarak M, Mu O, Singhasivanon P, White NJ and Nosten FH. Adverse effects of falciparum and vivax malaria and the safety of antimalarial treatment in early pregnancy: a populationbased study. The Lancet infectious diseases. 2012; 12(5):388-396.

44. Copple IM, Mercer AE, Firman J, Donegan G, Herpers B, 
Wong MH, Chadwick J, Bringela AD, Cristiano ML, van de Water B, Ward SA, O’Neill PM and Park BK. Examination of the cytotoxic and embryotoxic potential and underlying mechanisms of next-generation synthetic trioxolane and tetraoxane antimalarials. Molecular medicine. 2012; 18:1045-1055.

45. Finaurini S, Ronzoni L, Colancecco A, Cattaneo A, Cappellini MD, Ward SA and Taramelli D. Selective toxicity of dihydroartemisinin on human CD34+ erythroid cell differentiation. Toxicology. 2010; 276(2):128-134.

46. Orlowski RZ. Why proteasome inhibitors cannot ERADicate multiple myeloma. Cancer cell. 2013; 24(3):275-277.

47. Chapman MA, Lawrence MS, Keats JJ, Cibulskis K, Sougnez C, Schinzel AC, Harview CL, Brunet JP, Ahmann GJ, Adli M, Anderson KC, Ardlie KG, Auclair D, Baker A, Bergsagel PL, Bernstein BE, et al. Initial genome sequencing and analysis of multiple myeloma. Nature. 2011; 471(7339):467-472.

48. Gupta PB, Onder TT, Jiang G, Tao K, Kuperwasser C, Weinberg RA and Lander ES. Identification of selective inhibitors of cancer stem cells by high-throughput screening. Cell. 2009; 138(4):645-659.

49. Lee JJ, Kong M, Ayers GD and Lotan R. Interaction index and different methods for determining drug interaction in combination therapy. Journal of biopharmaceutical statistics. 2007; 17(3):461-480.

50. Chou TC. Drug combination studies and their synergy quantification using the Chou-Talalay method. Cancer research. 2010; 70(2):440-446.

51. Plummer JL and Short TG. Statistical modeling of the effects of drug combinations. Journal of pharmacological methods. 1990; 23(4):297-309.

52. O'Brien MC and Bolton WE. Comparison of cell viability probes compatible with fixation and permeabilization for combined surface and intracellular staining in flow cytometry. Cytometry. 1995; 19(3):243-255.

53. Garg TK and Chang JY. 15-deoxy-delta 12, 14-Prostaglandin J2 prevents reactive oxygen species generation and mitochondrial membrane depolarization induced by oxidative stress. BMC pharmacology. 2004; 4:6.

54. Botto S, Streblow DN, DeFilippis V, White L, Kreklywich CN, Smith PP and Caposio P. IL-6 in human cytomegalovirus secretome promotes angiogenesis and survival of endothelial cells through the stimulation of survivin. Blood. 2011; 117(1):352-361. 